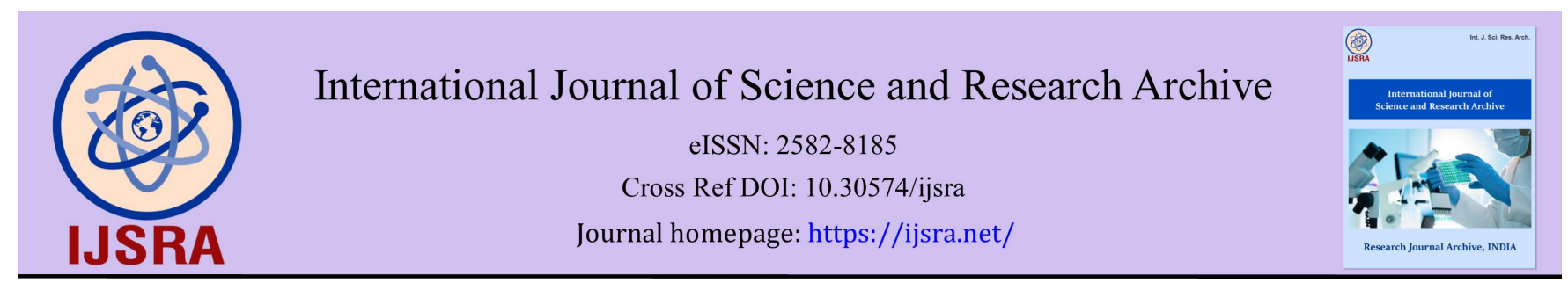

(RESEARCH ARTICLE)

Check for updates

\title{
Study of the outcome of pediatric patients with aplastic anemia treated with immunosuppressive therapy-Single center experience
}

\author{
Mohamed $\mathrm{W}^{*}$, Morsy N and Matbouly S \\ Pediatrics Hematology, Oncology and BMT, Faculty of Medicine, Ain Shams University, Cairo, Egypt.
}

International Journal of Science and Research Archive, 2021, 03(01), 097-106

Publication history: Received on 06 July 2021; revised on 12 August 2021; accepted on 14 August 2021

Article DOI: https://doi.org/10.30574/ijsra.2021.3.1.0116

\begin{abstract}
Background: Immunosuppressive therapy is the Initial therapy for children with severe Aplastic anemia (SAA) and lacking a matched related donor. Cyclosporine A (Cy A) alone has been tried as single agent in resource poor Countries.

Patients and Methods: This retrospective study was done to assess the outcome of immunosuppressive therapy (IST) in SAA children. It included 23 patients treated at Ain Shams University Pediatrics Hospitals between 2011 - 2019 with IST, they received rabbit anti-thymocyte globulin (r ATG) plus (Cy A) (Group I, 8 patients) or Cy A alone (Group II, 15 patients). The median follow- up duration was 59 months.

Results: one patient (4.3\%) developed AML clone. another patient (4.3\%) developed PNH clone. There was no significant difference in response between the two groups, Complete response was 34.7\%, 43.5\%, and $47.8 \%$ and partial response was $34.7 \%, 26.1 \%, 21.7 \%$ at 3,6, and 12 months, respectively in the whole cohort. NR was present in $30.4 \%$ at 3, 6, and 12 months. Hypertension in 62.5\% and higher levels of ALT, total and direct bilirubin were observed in group I as compared to group II. HAAA was clinically evident in 7 patients; 4 of them were hepatitis A, 1 was EBV, and 2 were seronegative for all viruses. At presentation they had significantly higher level of ALT, total bilirubin, and direct bilirubin.
\end{abstract}

Conclusion: IST is an effective therapy in patients with SAA with overall response of 70\%. Cy A alone can be an effective therapy in developing countries.

Keywords: Sever aplastic anemia; Immunosuppressive therapy; Aplastic anemia; Hepatitis associated; Outcome; Immunosuppressive therapy

\section{Introduction}

Acquired severe aplastic anemia (SAA) is a rare disorder characterized by peripheral bi- or pancytopenia and bone marrow (BM) hypoplasia [1]

According to the International Diagnosis Criteria for Aplastic Anemia, the disease can be divided into three types: Non SAA (NSAA), SAA and very SAA (VSAA) [2]. Initial therapy for younger patients with SAA is a matched sibling hematopoietic stem cell transplantation (HSCT) or immunosuppressive therapy (IST) if a matched sibling donor is not available [3]. Since the early 1990s, anti-thymocyte globulin (ATG) along with cyclosporine A (Cy A) have been considered the standard IST for AA patients, with an expected $50-60 \%$ probability of response and $60 \%$ overall survival at 1 year [4]. Children show higher rates of recovery and survival [2].

\footnotetext{
*Corresponding author: Mohamed W; E mail: drwafaa_ibrahim@med.asu.edu.eg

Pediatrics Hematology, Oncology and BMT, Faculty of Medicine, Ain Shams University, Cairo, Egypt. 
The beneficial effects of this regimen are believed to be due to the ability of the polyclonal antibodies in ATG to recognize a variety of human lymphocyte cell surface antigens, reduce lymphocyte numbers, and induce an immunosuppressive effect, which is further helped by the specific inhibitory effects of cyclosporine-A (Cy A) on T lymphocyte function [5].

Multiple studies have reported better outcomes with the use of horse ATG (h ATG) compared to rabbit ATG (r ATG) [5,6,7].

Cyclosporine-A alone has been tried as single agent in resource poor setting where patients cannot afford HSCT or ATG [6].

The response to IST is excellent and it should be offered as initial therapy prior to an alternative HSCT [7].

Nowadays with the global pandemic crisis of COVID 19, it is very challenging to offer proper immediate management for patients with severe aplastic anemia. Cy A alone may be an affordable therapeutic option [8].

This study was done to assess the response, predictors of response, outcome, and complications to IST in pediatric patients diagnosed with SAA and VSAA at Ain Shams University Pediatric Hospital.

\section{Material and methods}

\subsection{Patients}

This retrospective study included 23 pediatric patients diagnosed with SAA and VSAA at Pediatric Hematology and Transplantation Unit, Ain Shams University Hospitals. They received IST, either r ATG and Cy A (Group I) or Cy A alone (Group II) between January 2011 and 2019. The median follow-up duration was 59 months. Informed consent was obtained from Parents or guardians of all individual participants included in the study."

The diagnosis of AA was according to the International Diagnosis Criteria, including clinical manifestations, peripheral blood cell counts, and bone marrow hypoplasia, while excluding other hematopoietic diseases manifesting as pancytopenia.

Peripheral blood cell counts met at least two of the three following criteria: (1) hemoglobin (HB) <100 g/L; (2) platelets $<50 \times 10^{9} / \mathrm{L}$; (3) ANC < $1.5 \times 10^{9} / \mathrm{L}$. The severity of AA was classified in accordance with Camitta's criteria [9]. SAA was defined by meeting at least two of the three following criteria: (1) percentage of reticulocyte (Ret) $<1 \%$ or ARC $<20 \times$ $10^{9} / \mathrm{L}$; (2) ANC < $0.5 \times 10^{9} / \mathrm{L}$; (3) platelets $<20 \times 10^{9} / \mathrm{L}$. VSAA was considered if cases met the diagnostic criteria of SAA while ANC $<0.2 \times 10^{9} / \mathrm{L}[10]$. Patients with inherited bone marrow failure (IBMF), such as the Fanconi anemia (FA) and congenital dyskeratosis (DC) were excluded by testing chromosomal breakage and also CD 55 CD 59 to exclude PNH at initial diagnosis.

All patients had viral screen at diagnosis including hepatitis $A, B, C, E B V, C M V$, and $H S V$.

All patients with AA had HLA match at diagnosis. Patients with matched related donor (MRD) proceeded to hematopoietic stem cell transplantation. Patients with no available matched donor were planned for IST ( $\mathrm{r}$ ATG and Cy A) or Cy A alone according to the availability of cabinet and insurance coverage.

\subsection{Methods}

Data of the 23 patients were retrieved from the patients' files. They were 8 patients who received r ATG and Cy A (Group I) and 15 patients who received Cy A alone (Group II). The data included patients' demographics, detailed history and clinical examination, diagnostic workup for AA, immunosuppressive therapy protocol, assessment of the response at 3 months, 6 months and at 1 year, complications, and clonal evolution.

The ATG used was rabbit ATG-Fresenius (20 mg/kg/d)) IV for 4 consecutive days. In both groups, Cy A was given IV at a dose of $6 \mathrm{mg} / \mathrm{kg} / \mathrm{d}$ with a target level of $300 \mathrm{ng} / \mathrm{m}$. Cy A was continued orally for at least 6 months.

Determination of the response to IST was done according to Camitta's criteria [9]. Complete response (CR) was defined as $\mathrm{Hb}$ normal for age, ANC $>1.5 \times 10^{9} / \mathrm{L}$, and platelet count $>150 \times 10^{9} / \mathrm{L}$. Partial response (PR) was defined as transfusion independence associated with no longer meeting criteria for severe disease. Still severe or worse was considered no response (NR). Both CR and PR were considered as a positive response. 
Patients in Group I who failed to respond received a 2nd IST course after 6 months.

\subsection{Statistical Methods}

Statistical analysis was done using SPSS 20.0. Differences among variables were evaluated by the $\chi^{2}$ test (or Fisher's exact test for cell frequencies less than 5) and a standard t-test was used for continuous variables.

\section{Results}

This study included 23 pediatric patients with SAA and VSAA. Patients' characteristics are presented in table 1. The median age was 9 years, (IQR: 5 -13). Seventeen (73.9\%) were males and 6 (26.1\%) were females. Hepatitis associated aplastic anemia (HAAA) was a presentation in 7 patients (30.4\%), 4 of them were hepatitis $A$ virus (HAV) infection, 1 was $E B V$ infection, and the remaining 2 were seronegative. Repeated severe bacterial infection before treatment was present in 9 patients (39.1\%) and positive blood cultures among those patients were MRSA, E. Coli, Gram Negative bacilli, and acentobacter bacilli. Fungal infection before treatment occurred in 6 patients (26.1\%), most of them were candida, two were aspergillus.

Table 1 Characteristics of the patients

\begin{tabular}{|c|c|c|c|}
\hline \multirow{2}{*}{ Age (year) } & Median (IQR) & \multicolumn{2}{|c|}{$9(5-13)$} \\
\hline & Range & \multicolumn{2}{|c|}{$1-15$} \\
\hline \multirow{2}{*}{ Sex } & Male & \multicolumn{2}{|c|}{$17(73.9 \%)$} \\
\hline & Female & \multicolumn{2}{|c|}{$6(26.1 \%)$} \\
\hline \multirow{2}{*}{$\begin{array}{l}\text { Interval between diagnosis and } \\
\text { starting treatment (months) }\end{array}$} & Median (IQR) & \multicolumn{2}{|c|}{$2(1-5)$} \\
\hline & Range & \multicolumn{2}{|c|}{$1-36$} \\
\hline \multirow{2}{*}{ HAAA } & No & \multicolumn{2}{|c|}{$16(60.6 \%)$} \\
\hline & Yes & \multicolumn{2}{|c|}{$7(30.4 \%)$} \\
\hline & & No. & $\%$ \\
\hline \multirow{2}{*}{ Autoimmune markers } & No & 20 & $87.0 \%$ \\
\hline & ASMA positive & 3 & $13.0 \%$ \\
\hline \multirow{2}{*}{ Occurrence of leukemia } & No & 22 & $95.7 \%$ \\
\hline & Yes & 1 & $4.3 \%$ \\
\hline \multirow{2}{*}{ PNH Clonal evolution } & No & 22 & $95.7 \%$ \\
\hline & Yes & 1 & $4.3 \%$ \\
\hline
\end{tabular}

Table 2 Response to Therapy in All Patients

\begin{tabular}{|l|c|c|c|}
\hline & NR & PR & CR \\
\hline 3 months & $7(30.4 \%)$ & $8(34.7 \%)$ & $8(34.7 \%)$ \\
\hline 6 months & $7(30.4 \%)$ & $6(26.1 \%)$ & $10(43.5 \%)$ \\
\hline 12 months & $7(30.4 \%)$ & $5(21.7 \%)$ & $11(47.8 \%)$ \\
\hline \multicolumn{2}{|r|}{ NR: No }
\end{tabular}

Median time from diagnosis to treatment was 2 months with range of (1-5 months) for the entire cohort (table 1). Longer time was noted in Group I (10 months) than Group II (1month) (table 3). 
In Group I, one patient (4.3\%) developed acute myeloid leukemia (AML) after 19 months of CR. This patient did not respond to chemotherapy and died 3 months later. Another patient (4.3\%) developed PNH clone after 1 year of turning from CR to PR.

CR was $34.7 \%$ at 3 months, increased to $43.5 \%$ and $47.8 \%$ at 6 and 12 months respectively. PR was $34.7 \%$ at 3 months, decreased to $26.1 \%$ and $21.7 \%$ at 6 and 12 months respectively. NR was present in $30.4 \%$ at 3 months and stayed at the same percentage at 6 and 12 months (Table 2).

Table 3 Comparison between both groups

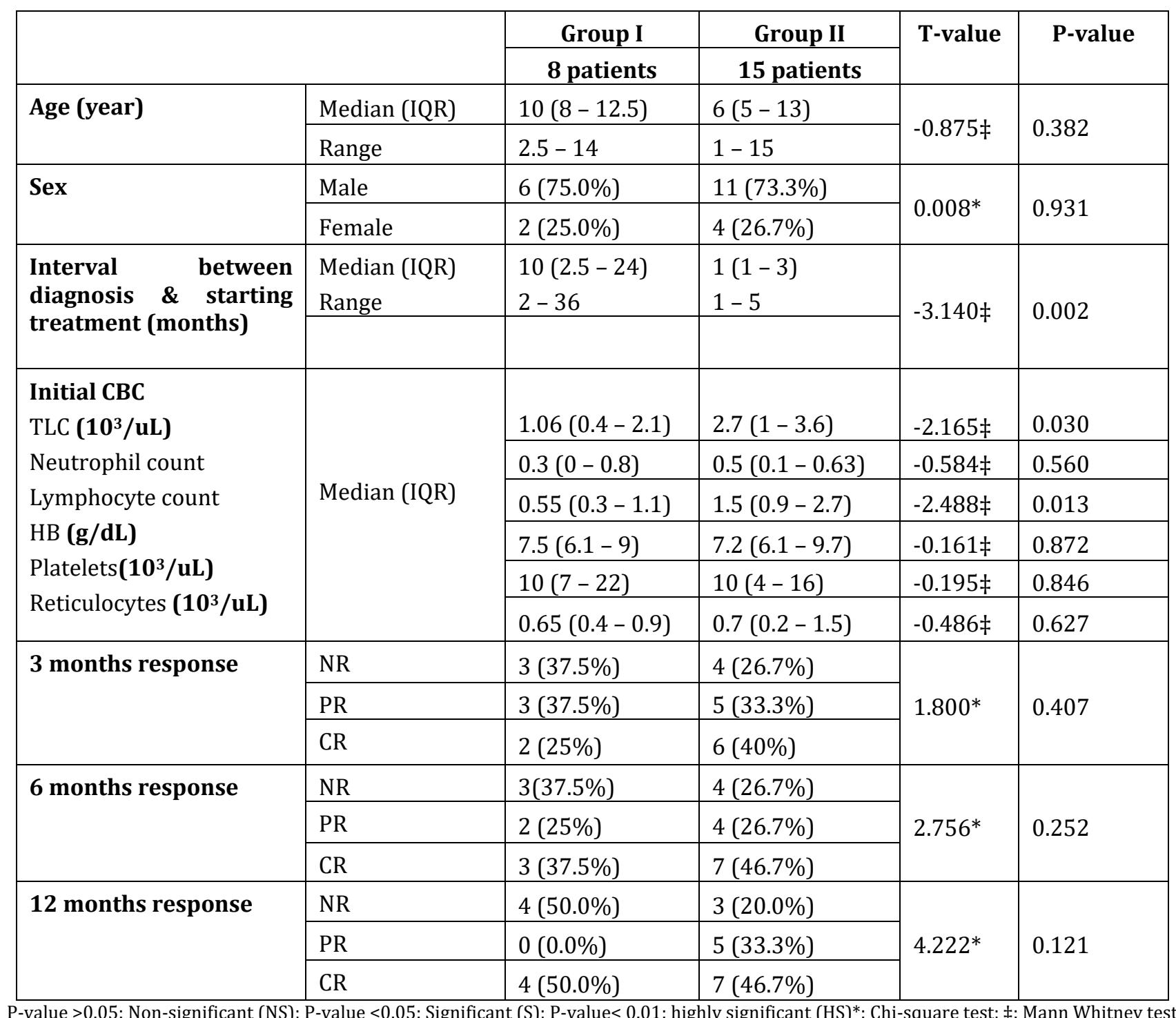

The interval between diagnosis and starting treatment was significantly longer in group I as compared to group II. The total leucocytic count and lymphocytic count were significantly higher in group II than group I. The response did not show statistically significant differences between the two groups at 3, 6, or 12 months (Table 3).

At 6 months, 3 patients in group I (37.5\%) had no response and received a $2^{\text {nd }}$ dose of IST. Those 3 patients still had NR at 12 months. All died later because of infections, bacterial or fungal. The fourth patient lost follow up. In group II, 3/15 patients (20\%) had NR at 12 months and were maintained on supportive care. 
Table 4 Predictors of response to immunosuppressive therapy

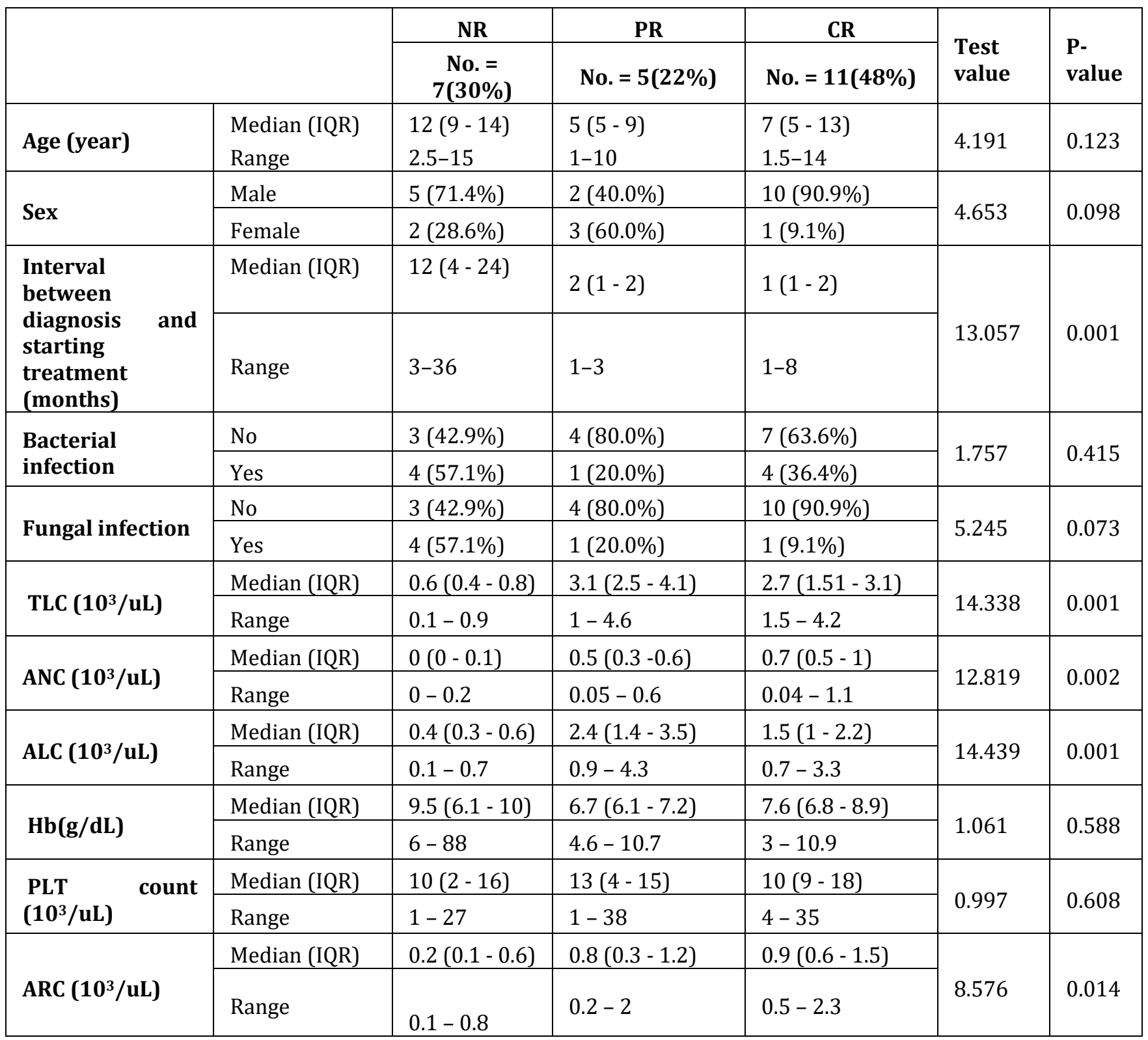

P-value $>0.05$ : Non-significant (NS); P-value $<0.05$ : Significant (S); P-value $<0.01$ : highly significant (HS)

‡: Kruskall Wallis test NR: No Response, PR: Partial Response, CR: Complete Response, TLC: Total Leucocytic Count, ANC: Absolute Neutrophil Count, ALC: Absolute Lymphocytic Count, PLT: Platelets, ARC: Absolute Reticulocytic Count

The interval between diagnosis and starting treatment showed high positive significant impact on the response to immunosuppressive therapy (P-value 0.001), the shorter the duration the better the response. Patients with initial high TLC, neutrophil count, lymphocyte count and absolute reticulocyte count had a significantly better response than those who had lower counts.

The level of ALT, total bilirubin, and direct bilirubin were significantly higher in HAAA group as compared to IAA group. Frequency of PRBCS transfusion before treatment was significantly better in HAAA group as compared to IAA group. Significant higher male to female ratio in HAAA and IAA groups. Four of the 7 patients (57\%) achieved complete ALT recovery prior to the diagnosis of aplastic anemia (table 5). 
Table 5 Comparison between idiopathic aplastic anemia (IAA) and hepatitis-associated aplastic anemia (HAAA) patients

\begin{tabular}{|c|c|c|c|c|c|c|c|c|}
\hline & \multirow{2}{*}{\multicolumn{2}{|c|}{$\begin{array}{c}\text { IAA } \\
\text { No. = } 16\end{array}$}} & \multirow{2}{*}{\multicolumn{2}{|c|}{$\begin{array}{l}\text { HAAA } \\
\text { No. }=7\end{array}$}} & \multirow{2}{*}{$\begin{array}{l}\text { Test } \\
\text { value }\end{array}$} & \multirow[t]{2}{*}{ P-value } & \multirow[t]{2}{*}{ Sig. } \\
\hline & & & & & & & & \\
\hline \multirow{2}{*}{ Age (year) } & Median (IQR) & \multicolumn{2}{|c|}{$7.5(5-12)$} & \multicolumn{2}{|c|}{$10(7-13)$} & \multirow{2}{*}{-1.006} & \multirow{2}{*}{0.314} & \multirow{2}{*}{ NS } \\
\hline & Range & \multicolumn{2}{|c|}{$1-15$} & \multicolumn{2}{|c|}{$2.5-14$} & & & \\
\hline \multirow{2}{*}{ Sex } & Male & 11 & $(68.8 \%)$ & 6 & $(85.7 \%)$ & \multirow{2}{*}{0.727} & \multirow{2}{*}{0.394} & \multirow{2}{*}{ NS } \\
\hline & Female & 5 & (31.3\%) & 1 & $(14.3 \%)$ & & & \\
\hline \multirow{2}{*}{$\begin{array}{l}\text { Interval between } \\
\text { diagnosis and } \\
\text { starting } \\
\text { treatment } \\
\end{array}$} & Median (IQR) & \multicolumn{2}{|c|}{$2(1-8.5)$} & \multicolumn{2}{|c|}{$2(1-4)$} & \multirow{2}{*}{-0.450} & \multirow{2}{*}{0.653} & \multirow{2}{*}{ NS } \\
\hline & Range & \multicolumn{2}{|c|}{$1-36$} & \multicolumn{2}{|c|}{$1-8$} & & & \\
\hline \multirow{2}{*}{$\begin{array}{l}\text { Frequency of } \\
\text { PRBCS } \\
\text { transfusion } \\
\text { before treatment }\end{array}$} & Weekly & 11 & $68.8 \%$ & 1 & $14.3 \%$ & \multirow{2}{*}{5.789} & \multirow{2}{*}{0.016} & \multirow{2}{*}{$S$} \\
\hline & Monthly & 5 & $31.3 \%$ & 6 & $85.7 \%$ & & & \\
\hline \multirow{2}{*}{$\begin{array}{l}\text { Frequency of } \\
\text { PLTS transfusion } \\
\text { before treatment }\end{array}$} & Weekly & 15 & $93.8 \%$ & 7 & $100.0 \%$ & \multirow[b]{2}{*}{0.457} & \multirow[b]{2}{*}{0.499} & \multirow[b]{2}{*}{ NS } \\
\hline & Monthly & 1 & $6.3 \%$ & 0 & $0.0 \%$ & & & \\
\hline \multirow{2}{*}{ ALT } & Median (IQR) & \multicolumn{2}{|c|}{$15.5(11-25)$} & 1176 & $37-1359)$ & -3751 & 0000 & HS \\
\hline & Range & & 34 & & -1500 & 年. & 0.000 & 110 \\
\hline T hilirubin & Median (IQR) & 1.5 & $-1.75)$ & 13.4 & $2.9-15)$ & -3750 & 0000 & HS \\
\hline 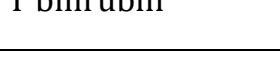 & Range & & -2 & & -34 & -0.150 & 0.000 & \\
\hline & Median (IQR) & 0.95 & $.6-1)$ & & $-11.5)$ & & & \\
\hline D bilirubin & Range & & -1.3 & & -25 & -3.766 & 0.000 & HS \\
\hline $\begin{array}{l}\text { Overall Response } \\
\text { months }\end{array}$ & to Therapy at 6 & & 5 \%) & & $1.4 \%)$ & $0.093^{*}$ & 0.954 & NS \\
\hline
\end{tabular}

Complications following initiation of IST such as hypertension, febrile neutropenia, hepatotoxicity, and gum hypertrophy were common. About $62.5 \%$ of patients in Group I were complicated by hypertension as compared with $20 \%$ of patients in Group II. Higher levels of ALT, total and direct bilirubin were observed in group I as compared to group II.

\section{Discussion}

Hematopoietic stem cell transplantation represents a curative treatment for idiopathic aplastic anemia. Its long-term survival rate was reported to be over $90 \%$ [1], but is substantially limited by the availability of human leukocyte antigenmatched donors. Therefore, in many cases, immunosuppressive therapy (IST) combining anti-thymocyte globulin (ATG) and cyclosporine A (Cy A) is used as a first-line treatment [11]. Although IST is a promising treatment option, the ability to predict its long-term outcomes remains poor due to refractoriness, relapses, and the risk of clonal evolution [12].

This work was done to study the response and predictors of response, outcome and complications to immunosuppressive therapy in children with severe and very severe aplastic anemia. Two groups were included; group I treated with (combined r ATG and Cy A) and group II treated with (Cy A alone). Hepatitis associated aplastic anemia (HAAA) was diagnosed in $30.4 \%$ of whole study population. 
The interval between diagnosis and starting treatment was significantly longer in group I than group II. In developing countries like Egypt, the availability of IST faces some difficulties because of high price of ATG, limited availability of cabinets to give the ATG, and the insurance coverage process that takes time. These factors may delay the start of ATG and sometimes limit it to a small number of patients.

Among the two groups, $43.5 \%$ had CR, 26.1\% had PR and 30.4\% had NR at 6 months. The positive response (CR + PR) to IST in this retrospective study was 70\%, indicating IST as an effective therapy for AA patients. The hematopoietic response rate of AA patients for IST is reportedly 42-74\%, with an overall long-term survival rate of approximately 90\% across several large studies in the United States, Europe, and East Asia [5-13]. Our results were similar to a study done by Gu et al. [14] that showed overall response of 77.66\%, a study done by Tichelli et al. [15] that was conducted on 192 patients, the overall response rate was $63 \pm 12 \%$, and also similar to a study done by Groarke et al. [16] where 28 $(72 \%)$ of all treated patients achieved a response at 6 months. Lower results were reported in a study done by Khemaphiphat et al. [17] where the 6-month overall response rates (ORRs) after the first and second courses of IST were $45.2 \%$ and $53.3 \%$, respectively.

The response to immunosuppressive therapy and long-term outcomes for pediatric severe aplastic anemia remains incompletely characterized. The difference in response could be attributed to the difference in the type of ATG used (horse or rabbit), difference in dose and duration together with the advance of supportive care in different areas [18].

In group II, the group which received cyclosporine A alone, $47.6 \%$ achieved complete response, while $33.3 \%$ had partial response and $20 \%$ had no response at 12 months. There was no death reported in this group all over the study period. In group I, $50 \%$ of patients had no response at 12 months, 3 of them died later and one lost follow up. A lower response was reported in an Indian study done by Shetty et al [6], where out of 16 patients who completed the study, complete response (CR) was observed in 3 patients, partial response (PR) in 6 patients, a total of 9 out of 16 (56\%) responded. Seven patients (44\%) did not respond. A greater response (76\%) at the end of 18 months was reported in a study by Hanif et al. [19]. Cyclosporine alone in low resource countries could help a group of children with no MRD and cannot afford the high cost combined ATG and Cyclosporine. The mortality rate in CyA alone group is lower than group I, an adding important point.

Shorter interval between diagnosis and initiation of IST was associated with a better response. The current study and similar studies highlighted the importance of early diagnosis and treatment in AA patients. Bacigalupo et al., [20] showed that the interval between diagnosis and treatment was the major predictor of response. Their results indicate that patients treated within 1 month after diagnosis might achieve the best response. Pediatric patients with AA often have atypical clinical manifestations due to their stronger bone marrow hematopoiesis potential than adults. Before typical pancytopenia occurs, they might only have reduction in one or two lineages of peripheral blood cells, which leads to difficulties in AA diagnosis.

Several predictive biomarkers for response to IST have been postulated, including age, gender, pre-treatment blood cell counts, cytokines, gene mutations, paroxysmal nocturnal hemoglobinuria and Telomere length (TL). But still the prognostic power of these reported biomarkers is currently insufficient to contribute to clinical decision making [12].

In the current study; higher initial TLC, neutrophil count, lymphocyte count and absolute reticulocyte count were associated with better response. These predictive factors for good response were confirmed in other studies and included younger age, higher ARC, higher ALC, and higher platelet counts [14,20].

The utility of ARC in predicting IST outcomes has been confirmed in a proportion of reports, but not in others [11,12,22].

Scheinberg et al., [7] found that ARC and ALC were predictive of response and survival in SAA patients treated with IST.

Further, the study of Japanese children demonstrated that lower white blood cell (WBC) count was indicative of a better response to IST rather than higher ANC [21].

Several studies were conducted to investigate the complications of treatment. Regarding the current study, all reported complications of febrile neutropenia followed by hypertension, elevated liver enzymes and gum hypertrophy were higher in group I than group II. Same complications were reported in all other studies using IST. Shah et al. [23] showed that febrile neutropenia was the most common (affecting 57.1\% patients) adverse event reported, followed by gum 
hypertrophy (15.4\%), hypertension (14.3\%), pneumonia, intra-cranial hemorrhage, and elevated creatinine level. None of the patients developed serum sickness.

The lower complications in group II (cyclosporine) alone are very encouraging to early start of this line of treatment in underdeveloped countries difficult to afford the cost burden of ATG.

In the current study, one Patient in group I (12.5\%) developed AML (4.3\% of the study population) 19 month after complete response. While another patient of the Group II (6.6\%) developed PNH clone at 1 year follow up (4.3\% of the study population).

Similarly, in the study done by Shah et al., [23] two patients (2.2\%) had clonal evolution-1 developed AML and died at 6 months after IST, the other developed acute lymphoblastic leukemia (ALL) at 7 months after IST after being in CR for 4 months. In another study by Kulasekararaj et al., [24], 11\% of patients under study progressed to MDS over median disease duration of 86 months (range, 9-260 months) and two patients of those evolved to MDS subsequently progressed to AML (1.3\% of the study population).

Development of cytogenetically abnormal clones or overt hematologic clonal disease was rare, although post-treatment marrow surveillance was not uniformly conducted in most of included studies [18]).

Clonal hematopoiesis $(\mathrm{CH})$ has recently been investigated more extensively using advanced genomics [25], which is an area very underdeveloped in our country. Genetic abnormalities were found in $50 \%$ of patients with AA in a study done by Ogawa et al., [25]. This was similar to a study done by Yoshizato et al., [26] in which clonal hematopoiesis was detected in $47 \%$ of the patients, most frequently as acquired mutations.

Prospective studies of late complications in AA identified an increased incidence of transformation to secondary MDS/AML in AA patients over time [27]. The incidence of secondary MDS/AML at 5 to 6 years after immunosuppressive therapy is estimated at $2 \%$ to $4 \%$, going up to $15 \%$ to $26 \%$ at 10 years of follow-up [28].

Hepatitis-associated aplastic anemia (HAAA) is a distinct variant of aplastic anemia in which bone marrow failure appears 2 to 3 months after an acute attack of hepatitis [29].

In the current study, 7 out of 23 patients (30.4\%) were HAAA. This percentage came higher than that reported by Wang et al., [30], who showed a prevalence of 3.8\% of HAAA among cases of SAA and Rauff et al. [29] who reported HAAA to constitute $4-10 \%$ of SAA cases in the Far East and 2-5\% in the West. The difference in prevalence might be related to the areas of different epidemiology of hepatitis. They were diagnosed clinically before pancytopenia occurred.

The diagnosis of HAAA in the current study was confirmed by clinical picture, laboratory results of elevated liver enzymes and hyperbilirubinemia. Pancytopenia developed 1-4 months after the attack of hepatitis. Four out of the seven cases were caused by hepatitis A virus, 2 had positive HAV IgM and the other 2 had positive HAV Ab several months later. One patient had Ebstein bar virus infection. The remaining 2 patients were seronegative for hepatitis viruses. In a previous study done by Patel et al., [31] hepatitis virus serology was negative in all patients with HAAA, while all patients had cholestatic hepatitis with sinusoidal obstruction morphology, and CD8+dominant lobular infiltrate by immunohistochemical staining. They suggested that HAAA may result from cytotoxic T-cell-mediated sinusoidal endothelial and hepatocytic injury.

The age of the HAAA patients in our study ranged from 2.5-14 years, with median age 10 years old. Six out of the seven patients were males (85.7\%). Significant higher male to female ratio was also reported by Safadi et al., [32].

There was nonsignificant difference in response to immune suppressive therapy between HAAA group and IAA which was $71.4 \%$ and $75 \%$ respectively at 6 months. Similar to the group of IAA. Gonzalez et al., [33] reported a response rate to IST of $70 \%$.

\section{Conclusion}

We have demonstrated that the overall response rate of IST in this retrospective study was 70\%, indicating that IST both ATG /Cy A or Cy A alone is an effective therapy for treating patients with AA especially in developing countries as 
an alternative therapy for children lacking MRD and or cannot afford the cost of ATG. Short duration to diagnoses and start of IST therapy was the major predictor of response in AA.

\section{Compliance with ethical standards}

\section{Disclosure of conflict of interest}

No conflict of interest.

\section{Statement of informed consent}

Statement of informed consent is written in patients and methods.

\section{References}

[1] Young NS, Bacigalupo A, Marsh JC. Aplastic anemia: pathophysiology and treatment. Biol Blood Marrow Transplant 2010; 16(1 Suppl): S119-125.

[2] Marsh JC, Ball SE, Cavenagh J, Darbyshire P, Dokal I, Gordon-Smith EC, et al. Guidelines for the diagnosis and management of aplastic anaemia. Br J Hematol. 2009; 147: 43-70.

[3] Bacigalupo A. How I treat acquired aplastic anemia. Blood. 2017; 129: 1428-1436.

[4] Damodar S. Immunosuppressive therapy for aplastic anemia. J Assoc Physicians India. 2015; 63: 16-203.

[5] Jeong DC, Chung NG, Cho B, Zou Y, Ruan M, Takahashi Y, et al. Long-term outcome after immunosuppressive therapy with horse or rabbit antithymocyte globulin and cyclosporine for severe aplastic anemia in children. Haematologica. Apr 2014; 99(4): 664-71.

[6] Shetty M, Narendra AM, Adiraju KP, Modugu NR. Study of aplastic anaemia with cyclosporine in resource poor setting. J Clin Diagn Res 2016; Jun;10(6): OC15-0C18.

[7] Scheinberg P, Wu CO, Nunez O, Young NS. Long-term outcome of pediatric patients with severe aplastic anemia treated with antithymocyte globulin and cyclosporine. J Pediatr. 2008; 153(6): 814-9.

[8] Terenziani M, Massimino M, Biassoni V, Casanova M, Chiaravalli S, Ferrari A, et al. SARS-CoV-2 disease and children under treatment for cancer. Pediatr Blood Cancer. 2020; e28346.

[9] Camitta BM, Storb R, Thomas ED. Aplastic anemia: pathogenesis, diagnosis, treatment, and prognosis. N Engl J Med. 1982; 306: 645-652.

[10] Bacigalupo A, Hows J, Gluckman E, Nissen C, Marsh J, Van Lint MT, et al. Bone marrow transplantation (BMT) versus immunosuppression for the treatment of severe aplastic anaemia (SAA): a report of the EBMT SAA working party. Br J Haematol. 1988; 70: 177-182.

[11] Miano M, Dufour C. The diagnosis and treatment of aplastic anemia: a review. Int J Hematol. 2015; 101(6): 52735.

[12] Narita A, Kojima S. Biomarkers for predicting clinical response to immunosuppressive therapy in aplastic anemia. Int J Hematol. 2016; 104(2), 153-158.

[13] Locasciulli A, Oneto R, Bacigalupo A, Socie G, Korthof E, Bekassy A, et al. Outcome of patients with acquired aplastic anemia given first line bone marrow transplantation or immunosuppressive treatment in the last decade: a report from the European Group for Blood and Marrow Transplantation (EBMT). Haematologica 2007; 92(1): 11-18.

[14] Gu C, Zhu X, Qiao X, Zhai X, Shi W, Xie X. Multivariate logistic analysis of predictors of response to immunosuppressive therapy in children with aplastic anemia: a double-center study. Hematology. 2019; 24(1): 282-289.

[15] Tichelli A, Schrezenmeier H, Socie G, Marsh J, Bacigalupo A, Duhrsen U, et al. A randomized controlled study in patients with newly diagnosed severe aplastic anemia receiving antithymocyte globulin (ATG), cyclosporine, with or without G-CSF: a study of the SAA Working Party of the European Group for Blood and Marrow Transplantation. Blood. 2011; 117, $4434-4441$. 
[16] Groarke EM, Patel BA, Diamond C, Gutierrez-Rodrigues F, Vicente A, Rios O, et al. Outcomes in Pediatric Patients with Severe Aplastic Anemia Treated with Standard Immunosuppression and Eltrombopag. Blood. 2019; 134: 454.

[17] Khemaphiphat P, Thedsawad A, Jianthanakanon J, Taka O, Wanachiwanawin W. Therapeutic response to immunosuppressive agents in Thai patients with transplant-ineligible aplastic anemia: possible predictive factors. J Hematol Transfus Med. 2017; 27(3): 251-260.

[18] Rogers ZR, Nakano TA, Olson TS, Bertuch AA, Wang W, Gillio A, et al. Immunosuppressive therapy for pediatric aplastic anemia: a North American Pediatric Aplastic Anemia Consortium study. Hematologica. 2019; Oct; 104(10): 1974-1983.

[19] Hanif S, Naz F, Siddiqui E, Raza J. Acquired Aplastic Anemia Treatment in a Developing Country. Pak J Med Sci. 2007; 23(3): 370-374.

[20] Bacigalupo A, Oneto R, Schrezenmeier H, Hochsmann B, Dufour C, Kojima S, et al. First line treatment of aplastic anemia with thymoglobuline in Europe and Asia: outcome of 955 patients treated 2001-2012. Am J Hematol. 2018; 93: 643-648.

[21] Yoshida N, Yagasaki H, Hama A, Takahashi Y, Kosaka Y, Kobayashi R, et al. Predicting response to immunosuppressive therapy in childhood aplastic anemia. Haematologica. 2011; 96(5): 771-4.

[22] Kulagin A, Lisukov I, Ivanova M, Golubovskaya I, Kruchkova I, Bondarenko S, et al. Prognostic value of paroxysmal nocturnal haemoglobinuria clone presence in aplastic anaemia patients treated with combined immunosuppression: results of two-centre prospective study. Br J Haematol. Feb 2014; 164(4): 546-54.

[23] Shah S, Jain P, Shah K, Patel K, Parikh S, Patel A, et al. Immunosuppressive therapy for aplastic anemia: a singlecenter experience from western India. Annals Hematol. 2019; 98(1): 41-46.

[24] Kulasekararaj AG, Jiang J, Smith AE, Mohamedali AM, Mian S, Gandhi S, et al. Somatic mutations identify a subgroup of aplastic anemia patients who progress to myelodysplastic syndrome. Blood. 23 Oct 2014; 124(17): 2698-2704.

[25] Ogawa S. Clonal hematopoiesis in acquired aplastic anemia. Blood. 2016; 128(3): 337-347.

[26] Yoshizato T, Dumitriu B, Hosokawa K, Makishima H, Yoshida K, Townsley D, et al. Somatic mutations and clonal hematopoiesis in aplastic anemia. N Eng J Med. 2015; 373(1): 35-47.

[27] Townsley DM, Scheinberg P, Winkler T, Desmond R, Dumitriu B, Rios O, et al. Eltrombopag added to standard immunosuppression for aplastic anemia. N Eng J Med. 2017; 376(16): 1540-1550.

[28] Babushok DV. A brief, but comprehensive, guide to clonal evolution in aplastic anemia. Hematol. 30 Nov 2018; 30(1): 457-466.

[29] Rauff B, Idrees M, Shah SA, Butt S, Butt AM, Ali L, et al. Hepatitis associated aplastic anemia: a review. Virol J 2011; 87(8): 1-6.

[30] Huaquan W, Meifeng Tu, Rong Fu, Yuhong Wu, Hong Liu, Xing L, et al. The clinical and immune characteristics of patients with hepatitis-associated aplastic anemia in China. PLOS ONE 9. 2014; 5.

[31] Patel KR, Bertuch A, Sasa GS, Himes RW, Wu H. Features of hepatitis in hepatitis-associated aplastic anemia: clinical and histopathologic study. J pediatr gastroenterol and nutrition 2017; 64(1): e7-e12.

[32] Safadi R, Or R, Ilan Y, Naparstek E, Nagler A, Klein A, et al. Lack of known hepatitis virus in hepatitis-associated aplastic anemia and outcome after bone marrow transplantation. Bone Marrow Transplant. 2001; 27(2): 183190.

[33] Gonzalez-Casas R, Garcia-Buey L, Jones EA, Gisbert JP, Moreno-Otero R. Systematic review: hepatitis-associated aplastic anemia-a syndrome associated with abnormal immunological function. Aliment Pharmacol Ther. 2009; 30(5): 436-443. 